\title{
Ação de capacitação de boas práticas de manejo e bem-estar animal aos profissionais do Turfe do Jockey Club de Pelotas
}

\author{
Training action of good management practices and animal welfare to Turf Professionals of the
} Jockey Club of Pelotas

Accíon de capacitación de buenas practicas del manejo y bien estar animal a los profesionales del Turfe del Jockey Club de Pelotas)

Taís Scheffer Del Pino

ORCID: https://orcid.org/0000-0003-4583-8280 Universidade Federal de Pelotas, Brasil

E-mail: tais.pino@gmail.com

Bruna da Rosa Curcio

ORCID: https://orcid.org/0000-0001-8678-3816 Universidade Federal de Pelotas, Brasil

E-mail: curciobruna@hotmail.com

Giovana Mancilla Pivato

ORCID: https://orcid.org/0000-0001-9139-3687 Universidade Federal de Pelotas, Brasil

E-mail: gimpivato@gmail.com

Margarida Aires da Silva

ORCID: https://orcid.org/0000-0002-6304-2465 Universidade Federal de Pelotas, Brasil

E-mail: guidaaires1@gmail.com

Gabriela Castro da Silda

ORCID: https://orcid.org/0000-0002-8555-2140 Universidade Federal de Pelotas, Brasil E-mail: gabicastrovini@gmail.com

Ruth Patten

ORCID: https://orcid.org/0000-0002-2593-1296 Francis Xavier University, Canadá

E-mail: ruthpatten@hotmail.com

Carlos Eduardo Wayne Nogueira

ORCID: https://orcid.org/0000-0002-8555-7953 Universidade Federal de Pelotas, Brasil

E-mail: cewnogueira@gmail.com

\begin{abstract}
Resumo
Este estudo traz a importância de ações educativas constantes aos profissionais do turfe do Jockey Club de Pelotas (JCP). O projeto de extensão "ClinEq - Grupo de Ensino, Pesquisa e Extensão em Medicina de Equino da Faculdade de Veterinária da UFPEL em parceria com o JCP realizou capacitação profissional com o objetivo de oferecer conhecimento e avaliar a percepção dos profissionais sobre boas-práticas de manejo com cavalos de corrida. Foram realizados seis encontros com exposição dos principais temas de manejo e três questionários referente a percepção dos treinadores em relação ao assunto abordado. Observou-se durante as capacitações que os profissionais demonstraram grande interesse nos conteúdos e aproveitaram todos os tópicos abordados, entendendo como um ganho na sua atuação profissional e melhorando seu entendimento sobre os conteúdos. Conseguindo durante o período que transcorreu a capacitação, colocar em prática algumas das sugestões apresentadas para melhorar as boas-práticas de manejo com os cavalos no JCP.
\end{abstract}

Palavras-chave: Nutrição; Odontologia; Estereotipia; Casqueamento; Antidoping.

\begin{abstract}
This study brings the importance of constant educational actions to the professionals of the Jockey Club de Pelotas (JCP). The extension project "ClinEq - Group Of Teaching, Research and Extension in Equine Medicine at the Veterinary Faculty of UFPEL in partnership with the JCP carried out professional training in order to offer knowledge and evaluate the professionals perception of good management practices with racehorses. Six meetings were held with an exposition of the main management themes and three questionnaires regarding the coaches perception in relation to the subject addressed. During the training sessions, it was observed that the participating professionals showed great
\end{abstract}


interest in the content and took advantage of all the topics covered, understanding it as a gain in their professional performance and improving their understanding of the content. Achieving, during the training period, put into practice some of the suggestions presented to improve good handling practices with horses at JCP.

Keywords: Nutrition; Dentistry; Stereotypes; Hoof trimming and shoeing; Anti-doping.

\section{Resumen}

Este estudio acerca la importancia de las acciones educativas constantes a los profesionales del Jockey Club de Pelotas (JCP). El proyecto de extensión “ClinEq - Grupo de Docencia, Investigación y Extensión en Medicina Equina de la Facultad de Veterinaria de la UFPEL en alianza con JCP realizó una formación profesional con el fin de ofrecer conocimiento y evaluar la percepción de los profesionales sobre las buenas prácticas de manejo con caballos de carreras. Se realizaron seis reuniones con exposición de los principales temas de gestión y tres cuestionarios sobre la percepción de los entrenadores en relación al tema abordado. Durante las capacitaciones se observó que los profesionales participantes mostraron gran interés por el contenido y aprovecharon todos los temas tratados, entendiéndolo como una ganancia en su desempeño profesional y mejorando su comprensión del contenido. Lograr, durante el período de entrenamiento, poner en práctica algunas de las sugerencias presentadas para mejorar las buenas prácticas de manejo con caballos en JCP.

Palabras clave: Nutrición; Odontología; Estereotipos; Corte de pezuñas y herrado; Antidopaje.

\section{Introdução}

Descobertas a respeito da inteligência, aprendizagem e emoções, dos animais, criaram novos conceitos e, consequentemente, mudanças de conduta em relação a eles (De Siqueira, 2020). Tais mudanças são embasadas na ciência do bem-estar animal, que compreende o estado da mente e do corpo do animal em expressar a sua naturalidade e estar na ausência de estados prolongados de medo ou estresse, satisfazendo características genéticas, fisiológicas, naturais e comportamentais da espécie e da raça (De Siqueira, 2020). Junior et al. (2012) descrevem, que essa ciência animal expõe as necessidades dos animais em áreas específicas, gerando uma alta produção de especificações técnicas na área do comportamento, manejo e bemestar animal e, consequentemente, aumento da demanda por profissionais que sejam capacitados para trabalhar inseridos nesses novos conceitos (Broom\&Molento, 2004).

Um dos segmentos considerado destaque dentro desse cenário é o Turfe. Segundo de Almeida (2020), dentre as modalidades esportivas equestres, a corrida é o esporte tradicional que mais envolve a criação, treinamento dos cavalos, competição e apostas que movimentam grandes quantias financeiras. E, além da geração de lucro, a produção e seleção de cavalos da raça Puro Sangue Inglês (PSI), também é objetivo desse esporte, pois esses possuem grande habilidade/aptidão na prática de corrida (Wilmsen, 2018). Contudo, principalmente devido ao aumento dessa prática esportiva nas grandes cidades, quanto melhor for o desempenho do equino, mais propenso está de passar a maior parte do seu tempo confinado, estando mais disposto a desenvolver lesões e alterações fisiológicas que afetam seu nível de bem-estar quando não corretamente manejado.

Outro ponto importante é que, no século 21, os métodos e formas de divulgação se ampliaram muito, permitindo que as informações rodassem em velocidade elevada pelo mundo e espalhando-se rapidamente. Essa modernização "líquida" dos meios de comunicação, junto à geração de novos conceitos de uma ciência em progresso, levou à rápida obsolescência técnica científica e assim, um aumento da demanda da atualização e capacitação profissional em todos os setores, inclusive no Turfe.

Em geral, os jóqueis iniciam suas carreiras profissionais na Escola Nacional de Profissionais ///.,ldo Turfe - ENPT, que desde 1950 ensina e treina técnicas de montaria para jovens que desejam competir e se tornar profissionais, correndo em hipódromos nacionais e internacionais (Labronici, 2016).

Com o fim da carreira como jóquei, estes profissionais tendem a se tornar treinadores, pois já estão neste meio e já aprenderam muito durante sua carreira. A partir de seus aprendizados na ENTP e com outros jóqueis e treinadores ao longo de sua carreia, o conhecimento empírico é adquirido muitas vezes dentro das próprias famílias. Estes profissionais acabam por se tornar treinadores no futuro, quando se aposentam como jóqueis. 
Ainda, apesar de fisicamente permanecerem fora das pistas, os treinadores são um dos personagens principais no turfe. Eles são os profissionais encarregados de preparar os animais para as carreiras e responsáveis, ao lado dos jóqueis, pelo bom desempenho nas corridas (Labronice, 2016). Porém, sua experiência e técnica é muito mais focada em como treinar e avaliar o cavalo na pista de corrida e muitas vezes os treinadores não aprendem ou se atualizam em relação a temas como a nutrição, bem-estar e manejo correto dos cavalos atletas com os quais eles trabalham. Por isso, se torna essencial a organização de eventos de capacitação para que possam adquirir conhecimento nesta área, pois é de fundamental importância para o desempenho e bem-estar do cavalo.

O objetivo do presente estudo foi capacitar os profissionais do turfe do Jockey Club de Pelotas através da abordagem pedagógica clássica com ações educativas na metodologia reuniões/palestras sobre temas voltados à saúde, manejo e bem-estar animal, assim como demonstrar a percepção dos mesmos perante os temas abordados.

\section{Metodologia}

Foi realizado no Jockey Club de Pelotas, durante o segundo semestre de 2020 (Setembro a Dezembro), a capacitação dos profissionais do Turfe sobre manejo e bem-estar com cavalos de corrida. A capacitação foi realizada em cinco palestras, com a duração de $2 \mathrm{~h}$ cada e com os seguintes temas: práticas que promovem o bem-estar do cavalo de corrida, nutrição do cavalo atleta, fisiologia do exercício, casqueamento e ferrageamento, odontologia equina e regramento para a utilização de medicamentos e antidopagem (Figura 1). Todas as atividades foram realizadas obedecendo aos protocolos sanitários e deliberações da Secretaria de Saúde do Município de Pelotas, dentro do plano de contingenciamento e distanciamento social Estado do Rio Grande do Sul, para enfrentamento a Pandemia de Covi-19.

$\mathrm{Na}$ capacitação foram realizados três questionários, visando entender e analisar o evento, assim como a didática do conteúdo abordado e a percepção dos profissionais sobre os principais temas. Estes possuíam uma média de nove questões de múltipla escolha e foram aplicados em momentos distintos da capacitação.

Ao final, foi obtida uma base de dados criada de forma manual em formato de planilha Excel $^{\circledR}$ e para as questões discursivas foi realizada correlação por semelhança e criada variáveis objetivas. Com isso, foi realizada análise estatística descritiva com indicação de frequência dos resultados por meio do software Statistix10.

Figura 1: Equipe de treinadores e palestrantes durante a capacitação realizada em 2020.

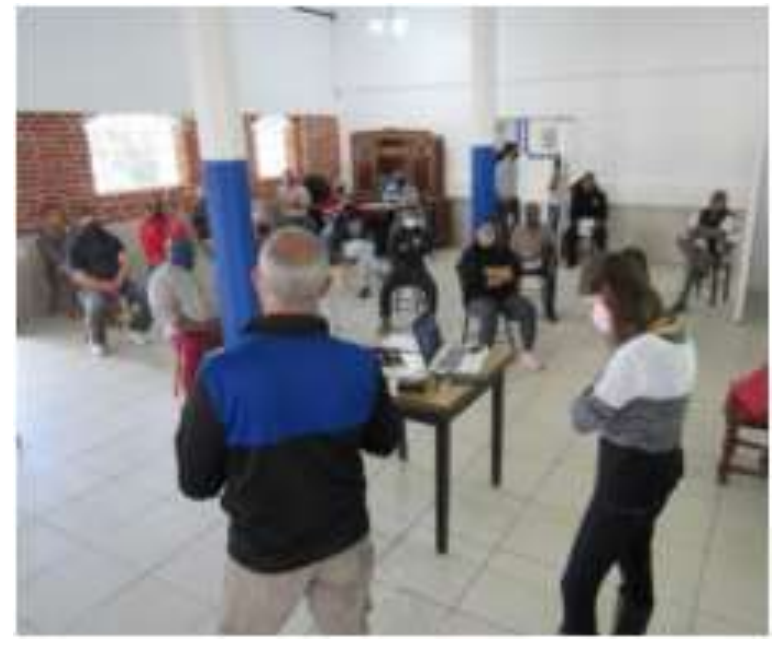

Fonte: Álvaro Guimarães. 


\section{Resultados}

A capacitação iniciou com um total de 24 profissionais do Turfe do Jockey Club de Pelotas, sendo este número mantido até o final da última palestra. O grupo de profissionais era composto na totalidade por treinadores, e as palestras foram ministradas em um espaço concedido dentro da cidade hípica e atendendo as normas de distanciamento social e higienização estabelecidas pela Secretaria de Saúde do Município de Pelotas, dentro do plano de contingenciamento e distanciamento social, Estado do Rio Grande do Sul e Organização Mundial da Saúde (OMS), para sua correta e segura realização.

Ao longo da capacitação foi observado o aumento do interesse dos profissionais pelos assuntos abordados, através de interações positivas com os palestrantes e a realização de perguntas. Houve também a aplicação de questionários como forma de observar a percepção dos mesmos, perante os conteúdos abordados e demais pontos da capacitação em seu entendimento e interesse, resultando em um total de 17 de 24 respostas.

A Figura 2 mostra o conhecimento dos treinadores antes e após a primeira palestra de maneira simplificada.

Figura 2: Avaliação do conhecimento dos treinadores sobre o bem-estar do cavalo de corrida antes e após a primeira palestra.

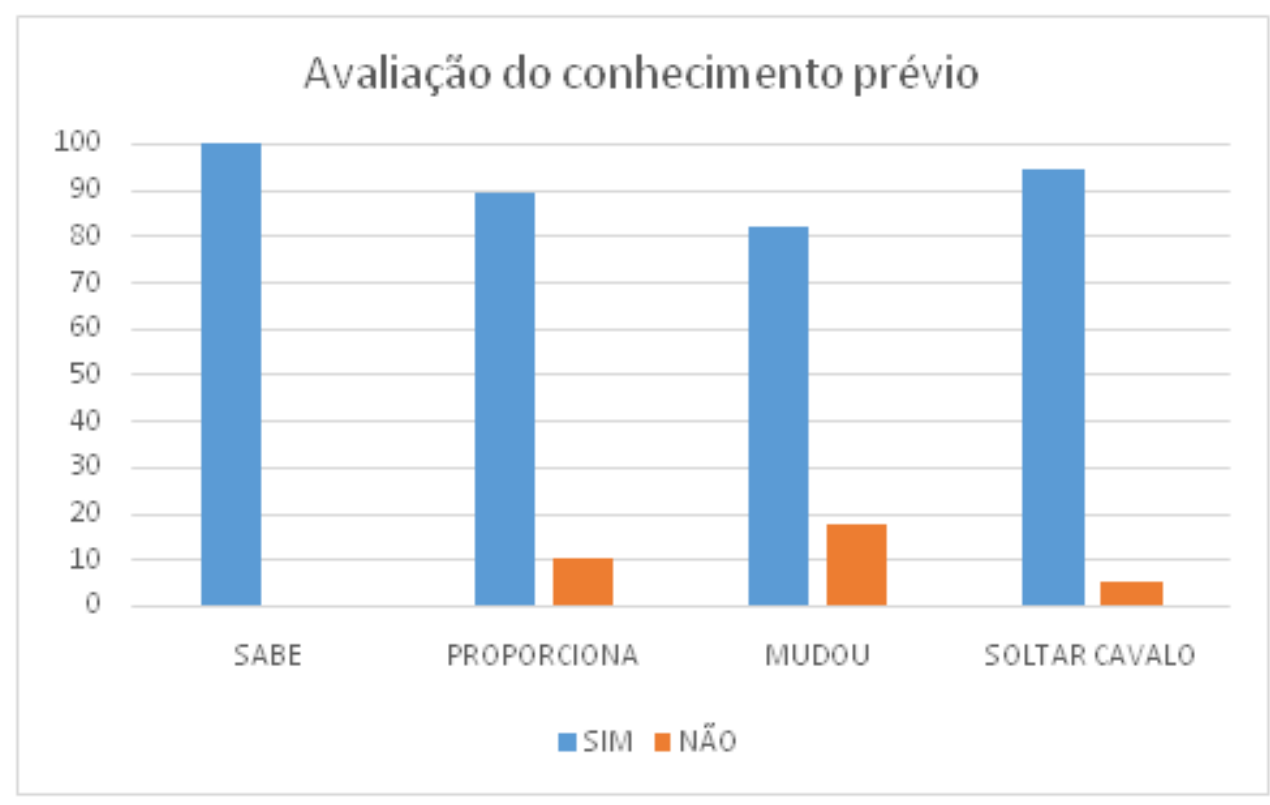

Fonte: Autores.

No primeiro questionário, os treinadores responderam sobre as estereotipias apresentadas pelos animais no Jockey Club de Pelotas, como mostrado abaixo na (Figura 3). 
Research, Society and Development, v. 10, n. 6, e56010615996, 2021

(CC BY 4.0) | ISSN 2525-3409 | DOI: http://dx.doi.org/10.33448/rsd-v10i6.15996

Figura 3: Avaliação do conhecimento dos treinadores sobre a presença de estereotipias em seus animais.

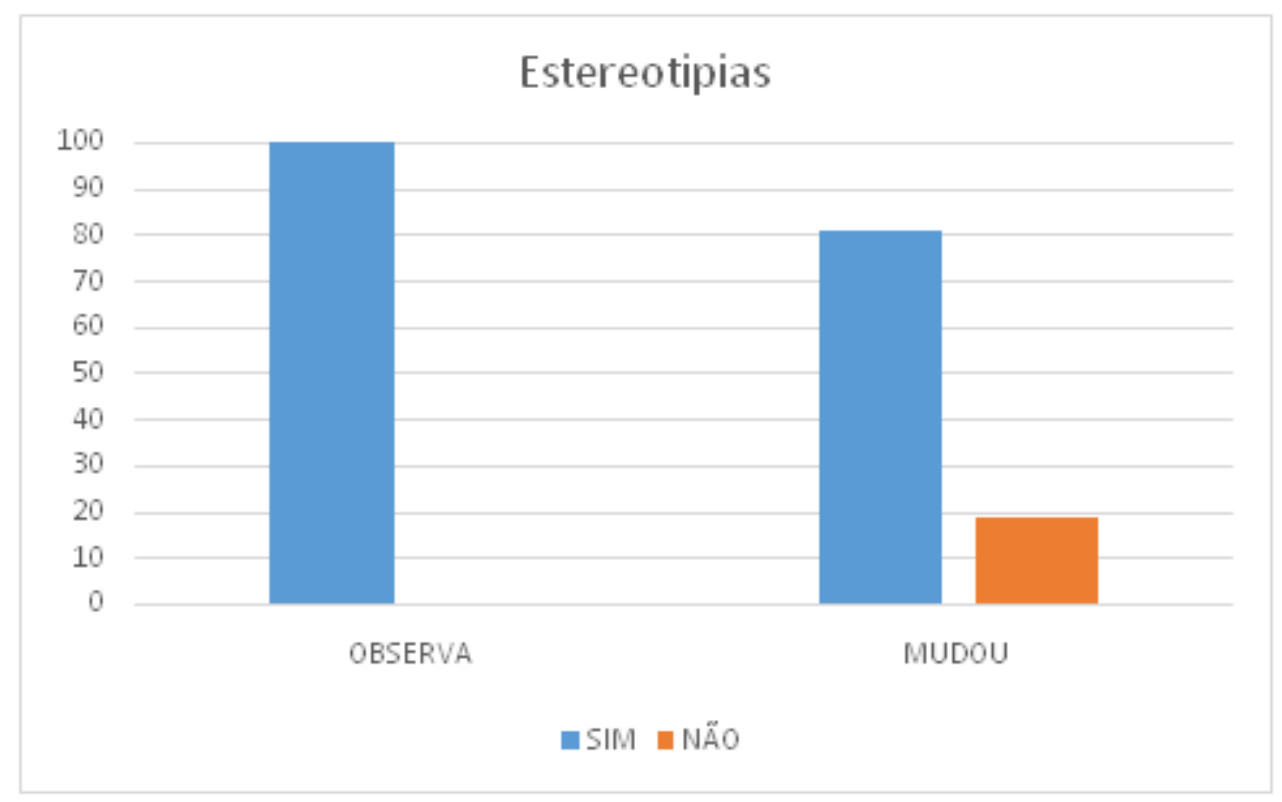

Fonte: Autores.

Também foi perguntado aos treinadores se o bem-estar animal pode interferir no resultado das corridas, como mostra a (Figura 4).

Figura 4: Avaliação da opinião dos treinadores sobre o bem-estar do cavalo interferir no resultado das corridas.

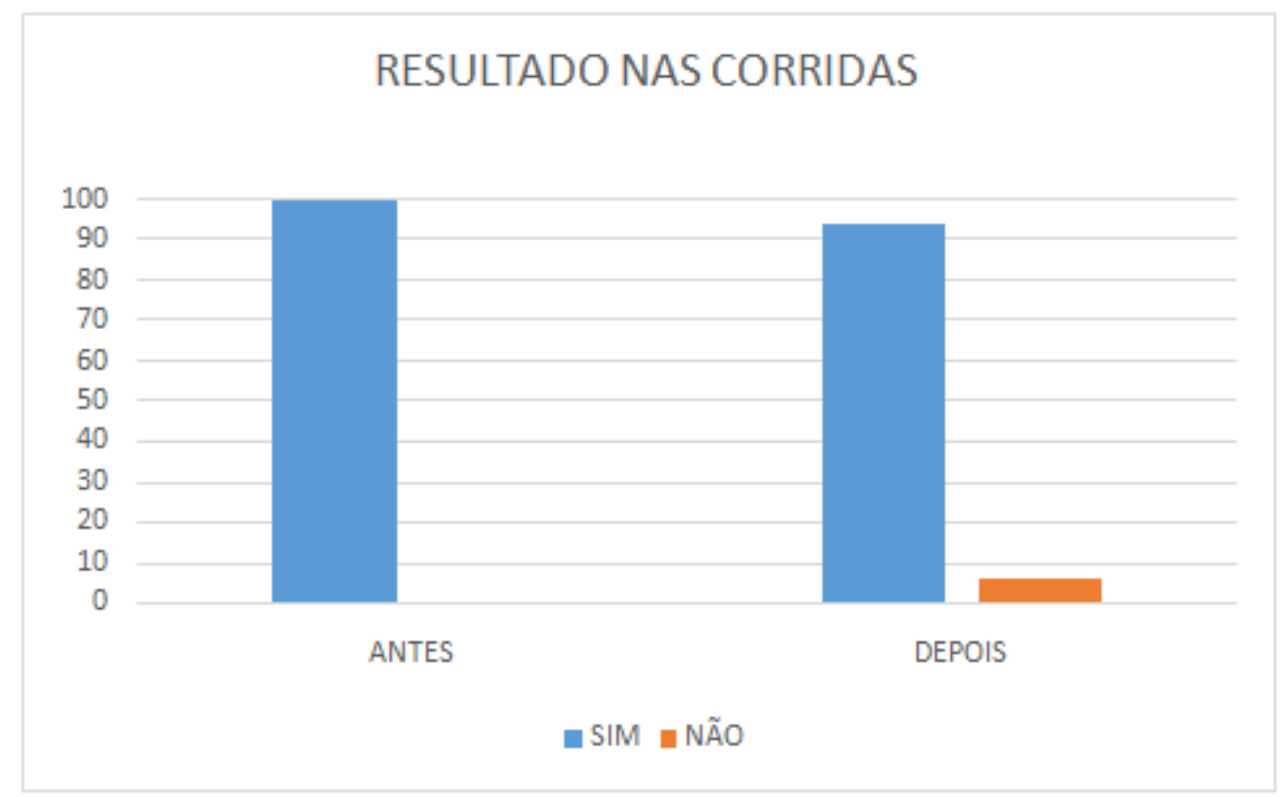

Fonte: Autores.

Os questionários 1 e 2, mostram amplamente a percepção dos treinadores perante as palestras apresentadas (Figura 5). 
Figura 5: Avaliação dos treinadores quanto a qualidade e interesse pessoal com a primeira palestra da capacitação.

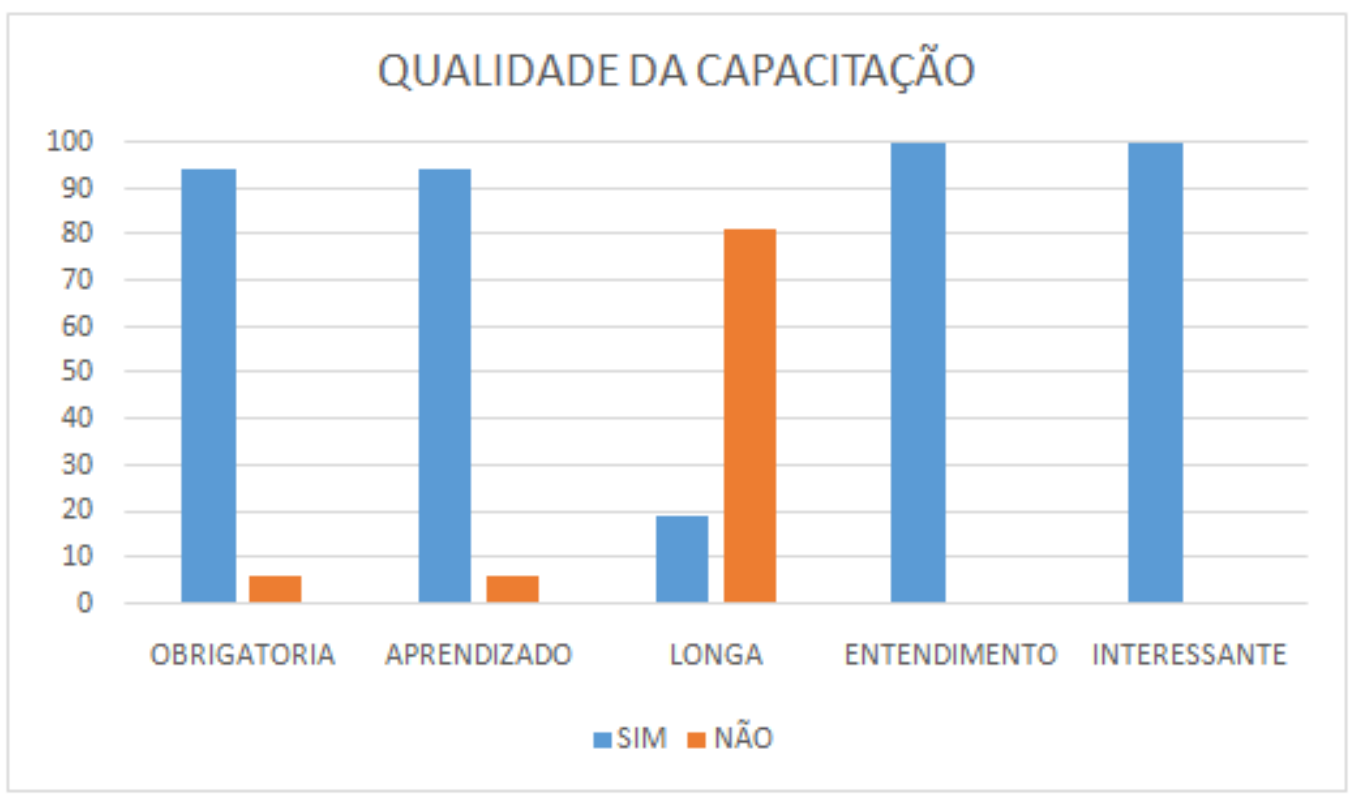

Fonte: Autores.

Visto os resultados apresentados na primeira etapa da avaliação, alguns pontos foram reafirmados com os treinadores após o final da capacitação, como por exemplo, o conhecimento dos mesmos em relação ao conceito de bem-estar animal e sobre proporcionarem o mesmo aos seus cavalos (Figura 6).

Figura 6: Visão e avaliação geral dos treinadores após todas as palestras. A. Você sabe o que é Bem-estar animal?; B. Você acredita que proporciona bem-estar ao seu cavalo?; C. Acrescentou ou retirou algo da alimentação dos cavalos após as capacitações?; D. Você utiliza treinamentos diferentes para cada cavalo(idade/distância)?; E. Seu entendimento sobre ferrageamento e casqueamento melhorou após a capacitação?; F. A capacitação mudou seu conceito sobre odontologia equina?; G. Acha que é importante o cuidado com a boca do cavalo?; H. Se esta capacitação não fosse obrigatória, você participaria?; I. Você achou as capacitações muito longas?; J. Você entendeu tudo que foi abordado?; L. Você achou o conteúdo apresentado interessante?

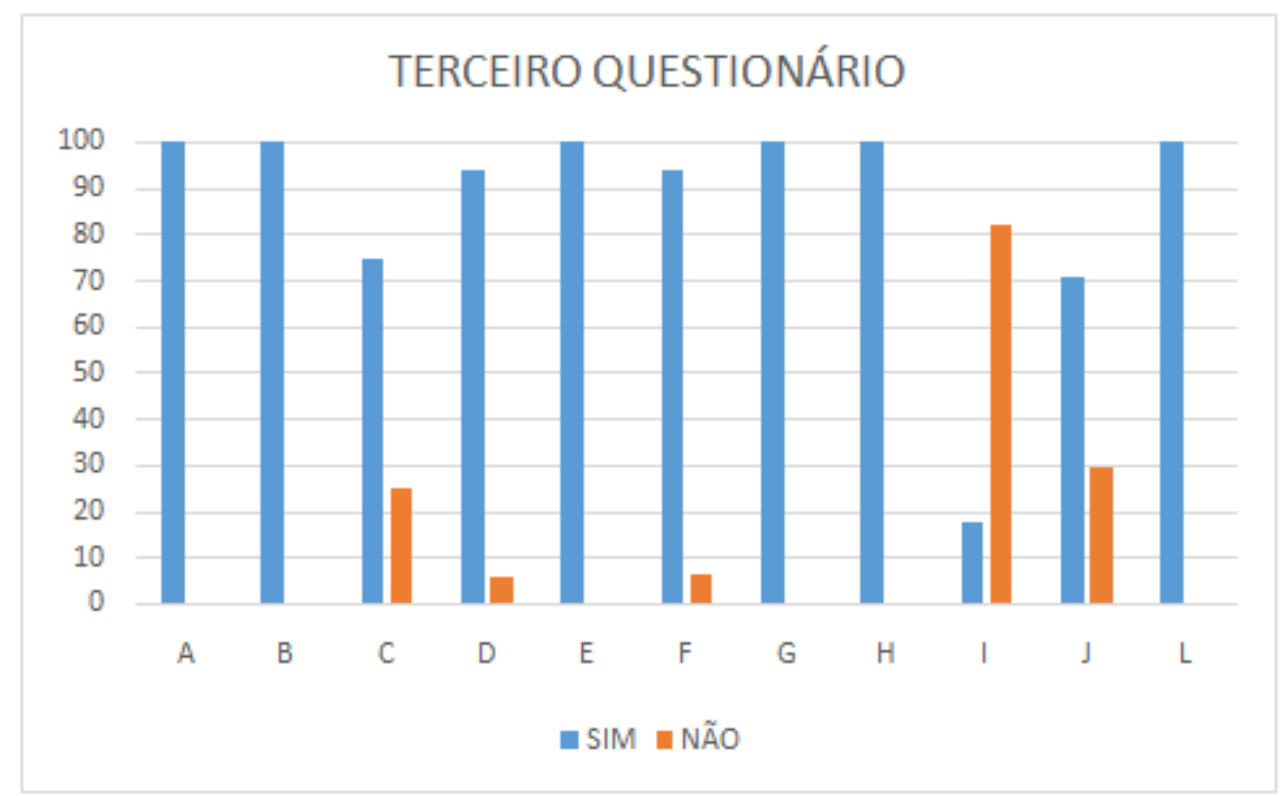

Fonte: Autores. 
Quando os profissionais foram questionados sobre a capacitação, responderam que puderam revisar conceitos de manejo, saúde e bem-estar animal, repensando suas práticas e aplicações no dia a dia com seus animais.

\section{Discussão}

Os profissionais que atuam como treinadores do Turfe, trabalham sob pressão. Além das exigências técnicas das competições, alcance de metas, busca por premiações, melhoria no treinamento e consequentemente o resultado em pista, treinam e são responsáveis por seres vivos sem expressão verbal de seus limites e afecções. Por este motivo, treinamentos realizados nesses moldes são extremamente importantes para garantir o bem-estar animal, assim como aperfeiçoar e otimizar a rotina de trabalho dos profissionais, aprimorando as técnicas conhecidas e instruindo a novos conceitos do comportamento e fisiologia equina. Segundo Simões, et all (2015) a formação, a prática e a capacitação profissional minimizaram o estresse ocupacional de profissionais de enfermagem no estudo citado, podendo se entender este resultado a outras áreas.

Dos profissionais que participaram da capacitação, realizada no Jockey Club de Pelotas, 94,1\% responderam que participariam até o final, mesmo se não fosse obrigatória. Estes também alegaram que aprenderam com as palestras (94,1\%), gerando interferências positivas nas suas rotinas de trabalho, atingindo a capacitação, então, seu propósito. Segundo Bucchi\& Mira (2010), o treinamento de profissionais é compreendido como um meio de aumentar o conhecimento teórico-prático que capacita o indivíduo para melhor realização de seu trabalho, o que amplia as competências profissionais e pessoais instrumentalizando o indivíduo para a transformação da realidade, indo ao encontro dos dados apresentados nesse trabalho.

O cavalo é um animal originalmente de vida social e livre, quando encachoeirado, o que compreende a maior parte do tempo dos cavalos do turfe, ele tende a estar com seu estado geral abalado e sob estresse (Beaver, 2019).

Essa alteração do estado emocional do animal, juntamente com o aumento do tempo de ócio causado pela estabulação podem levar a estereotipias. Até mesmo um manejo incorreto ou uma interação inapropriada podem ser uma causa de estresse que levem a uma alteração de comportamento (BROOM, 1993). Está definido que um confinamento de mais de 4 h por dia, pode ser um fator importante para o surgimento das estereotipias (Moretti, 2020) e essas foram notada pelos treinadores desta capacitação, uma vez que a (figura 3), 100\% deles (primeira coluna) alegaram que seus cavalos demonstram vícios/estereotipias, corroborando com os fatos apresentados na literatura. Contudo, nota-se também que os mesmos possuem preocupação quanto aos malefícios desses vícios ao animal, uma vez que 94,7\% deles proporcionam momentos de liberdade aos cavalos, fora dos momentos de treinamento e provas, e $81.3 \%$ deles utilizam algum tipo de enriquecimento ambiental com seus animais.

Leme e colaboradores em 2014 demonstraram que, o tipo de esporte para o qual o cavalo é utilizado deve determinar as atividades e as práticas de manejo aos quais ele está submetido. Por este motivo, é importante para o profissional que atue no turfe, o conhecimento das necessidades fisiológicas e naturais dos cavalos atletas, sempre aprendendo como aplicá-las buscando boas- práticas de manejo. Esta percepção ocorreu durante a capacitação (Figura 4), em que 100\% dos treinadores responderam que o bem-estar interferia no resultado das corridas, tendo apenas 5,9\% (1 resposta - terceira coluna) que mudou de opinião após a palestra.

Quanto às técnicas de ensino e aprendizagem junto ao manejo dos cavalos, 94,1\% dos entrevistados responderam que utilizam treinamentos diferentes para cada animal, principalmente levando em relação a idade e a distância percorrida. Ressalta-se que historicamente, os gregos eram observadores do comportamento do cavalo e empregavam a compreensão e entendimento deste no manejo e treinamento de seus animais, contudo os romanos não pareciam herdar as habilidades de cavalaria dos gregos, e muitos empregaram força e coerção no treinamento de cavalos, sem grande importância nas particularidades de cada animal (Waran, 2007). Contudo é sabido que, os animais treinados da primeira maneira eram mais 
fiéis aos seus cavaleiros e possuíam melhor desempenho nas batalhas (Waran, 2007). Este fato e conceito de treinamento histórico corrobora com a necessidade de entender o comportamento do cavalo e seus limites, o respeitando para o crescimento e melhor treinamento, apresentada pelos entrevistados.

Foi avaliado neste trabalho também o tempo de cada palestra e da capacitação como um todo, segundo Leme et Al., (2014), a escolha do tempo adequado para uma capacitação é fator influenciador do ensino e da aprendizagem do público alvo, possibilitando que os treinamentos venham a ser mais valorizados, identificados e assimilados pelos participantes como um método real de qualificação no trabalho. Visto que a maioria do público alvo $(81,2 \%)$ afirmou não ter sido muito longa a capacitação e terem entendido o conteúdo abordado, vemos, portanto, que o tempo utilizado para a duração das palestras e da capacitação como um todo foi adequado.

Ainda sobre o entendimento do conteúdo abordado, a linguagem utilizada em uma capacitação deve ser diversificada, para que o conteúdo descrito esteja acessível a múltiplos estilos de aprendizagem (Bicudo, 2011). É importante para o sucesso de toda capacitação usar a forma de linguagem adequada ao público alvo, uma vez que as utilizações de termos não apropriados podem levar a interpretação errônea da informação e, consequentemente, ao déficit do aprendizado. Neste trabalho vimos que apesar de bem-sucedida a capacitação como um todo, praticamente trinta por cento dos treinadores não entenderam completamente os conteúdos abordados, mostrando que em um próximo momento outras metodologias de ensino e linguagens podem ser utilizadas e exploradas para o público em questão.

Ressalta-se por fim, que a capacitação se passou em um ano de pandemia mundial do Covid-19, o que pode ter influenciado em diversos pontos da mesma, contudo mesmo com todas as adversidades do momento, principalmente o distanciamento social, desinfecção das mãos e o uso da máscara de proteção, 100\% dos entrevistados avaliaram como positiva, interessante e fundamental a capacitação, participando em sua totalidade mesmo se não fosse obrigatória e aplicando a mesma direta ou indiretamente no dia a dia dos seus animais.

\section{Conclusão}

A percepção dos treinadores sobre boas-práticas de manejo com cavalos de corrida melhorou significativamente com a capacitação. As técnicas e conhecimento dos mesmos foram aprimoradas e novos conceitos do comportamento e fisiologia equina foram adquiridos. Conclui-se que as palestras geram interferências positivas na rotina de trabalho dos treinadores e que as capacitações atingiram seu propósito. Sendo assim, é necessário que as capacitações sigam ocorrendo a cada ano, para que o conhecimento se propague e as boas práticas de manejo com cavalos de corrida evolua sempre, junto com os cavalos e com os treinadores, gerando assim mais trabalhos sobre este assunto tão importante.

\section{Referências}

Bicudo, S. F., \& Rodrigues, B. C. M. (2011). Oficina para Capacitação de Professores Conteudistas. Octavo Simposium Iberoamericano en Educación, Cibernética e Informática.

Broom, D. M., \& Kennedy, M. J. (1993). Stereotypies in horses: their relevance to welfare and causation. Equine Veterinary Education, 5, 151-151.

Broom, D. M., \& MOLENTO, C. F. M. (2004). Bem-estar animal: Conceito e Questões relacionadas revisão. Archives of veterinary Science, 9(2).

Bucchi, S. M., \& Mira, V. L. (2010). Reelaboração do treinamento admissional de enfermeiros de Unidade de Terapia Intensiva. Revista da Escola de Enfermagem da USP, 44(4), 1003-1010.

de Almeida, C. R. F., de Souza, A. D. S., dos Santos Filho, M., \& Botteon, P. D. T. L. (2020). Avaliação de arritmias em cavalos atletas com baixo desempenho. Research, Society and Development, 9(9), e425997148-e425997148.

de Siqueira, V. C., \& de Santis Bastos, P. A. (2020). Bem-estar animal para clínicos veterinários. Brazilian Journal of Health Review, 3(2), 1713-1746.

Gontijo, L. A., Cassou, F., Duarte, P. C., Lago, L. A., Alves, G. E., Melo, M. M., \& Faleiros, R. R. (2018). Bem-estar em equinos do Jockey Club do Paraná: indicadores clínicos, etológicos e ritmo circadiano do cortisol. Pesquisa Veterinária Brasileira, 38(9), 1720-1725. 
Research, Society and Development, v. 10, n. 6, e56010615996, 2021

(CC BY 4.0) | ISSN 2525-3409 | DOI: http://dx.doi.org/10.33448/rsd-v10i6.15996

Junior, A., Bergmann, M., \& Silva, N. (2012, December 11). Bem estar animal. https://vet.ufmg.br/ARQUIVOS/FCK/file/editora/caderno tecnico 67 Bem Estar Animal ok.pdf

Labronici, R. B. (2016). A pureza do sangue hibrido: os bastidores do turfe para a produção de cavalos e homens de corrida. ILUMINURAS, 17(42).

Leme, D. P., Parsekian, A. B. H., Kanaan, V., \& Hötzel, M. J. (2014). Management, health, and abnormal behaviors of horses: A survey in small equestrian centers in Brazil. Journal of Veterinary Behavior, 9(3), 114-118.

Leme, D. P., Silva, E. L., Vieira, M. C., \& Buss, L. P. (2017). Manual de Boas-Práticas de Manejo em Equideocultura. Brasil. Ministério da Agricultura, Pecuária e Abastecimento. Secretaria de Mobilidade Social, do Produtor Rural e Cooperativismo. Brasília: MAPA/ACE/CGCS, 50p.

Maio Ysa, C. B. (2020). Impacto de la rotación de personal en el cumplimiento de objetivos de la tienda Sodimac del Jockey Plaza.

Moretti, M., \& Perrone, M. (2020). El bienestar animal en equinos según el modelo IPAE. Calidad de Vida y Salud, 13(Especial), 184-194.

Quadros, J.; Molento, C. F. M.; Ensino de bem-estar animal para médicos-veterinários noBrasil: atualização 2008. In: Congresso Brasileiro de Medicina Veterinária, 35, 2008, Gramado.

Silva, A. (2017, December). Aspectos de Princípios da Doma Racional na Conduta Hospitalar Visando o Bem-Estar dos Equinos - Revisão de Literatura. http://www.cstrold.sti.ufcg.edu.br/grad_med_vet/tcc_2016.2/27_amanda_de_araujo_silva.pdf

Simões, J. S., Otani, M. A. P., \& Júnior, A. C. S. (2015). Estresse dos profissionais de enfermagem em uma unidade de urgência/Stress of the nursing professionals in an emergency unit. REGRAD-Revista Eletrônica de Graduação do UNIVEM-ISSN 1984-7866, 8(1), 75-95.

Ribeiro, L. C. (2020). Bem estar e desempenho do cavalo atleta.

Waran, N. (Ed.). (2007). O bem-estar dos cavalos (Vol. 1). Springer Science \& Business Media.

Wilmsen, M. O. (2018). Avaliação da tromboelastometria (Rotem® Delta) em cavalos de turfe da raça puro sangue inglês com hemorragia pulmonar induzida pelo exercício. 